\title{
First results on low-mass WIMP from the CDEX-1 experiment at the China Jinping underground Laboratory
}

\author{
W. Zhao, ${ }^{1}$ Q. Yue, ${ }^{1, \text { * }}$ K.J. Kang, ${ }^{1}$ J.P. Cheng,,${ }^{1}$ Y.J. Li, ${ }^{1}$ S.T. Lin,,${ }^{7}$ 丹 Y. Bai, ${ }^{3}$ Y. Bi,${ }^{5}$ J.P. Chang, ${ }^{4}$ N. Chen, ${ }^{1}$ N. \\ Chen, ${ }^{1}$ Q.H. Chen, ${ }^{1}$ Y.H. Chen,${ }^{6}$ Y.C. Chuang, ${ }^{7}$ Y Z. Deng, ${ }^{1}$ C. Du ${ }^{1}$ Q. Du,${ }^{1}$ H. Gong, ${ }^{1}$ X.Q. Hao, ${ }^{1}$ H.J. He, ${ }^{1}$ Q.J. \\ He, ${ }^{1}$ X.H. Hu, ${ }^{3}$ H.X. Huang, ${ }^{2}$ T.R. Huang, ${ }^{7,}{ }^{1}$ H. Jiang, ${ }^{1}$ H.B. Li, ${ }^{7}$, J.M. Li, ${ }^{1}$ J. Li ${ }^{1}{ }^{1}$ J. Li ${ }^{4}{ }^{4}$ X. Li, ${ }^{2}$ X.Y. Li, ${ }^{3}$ Y.L. \\ Li, ${ }^{1}$ H.Y. Liao, ${ }^{7}$ 团 F.K. Lin, ${ }^{7}$ 过 S.K. Liu,${ }^{5}$ L.C. Lü, ${ }^{1}$ H. Ma, ${ }^{1}$ S.J. Mao, ${ }^{4}$ J.Q. Qin, ${ }^{1}$ J. Ren, ${ }^{2}$ J. Ren, ${ }^{1}$ X.C. Ruan, ${ }^{2}$

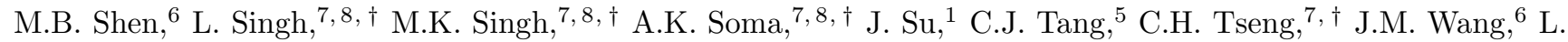 \\ Wang, ${ }^{5}$ Q. Wang, ${ }^{1}$ H.T. Wong, ${ }^{7}$ 由 S.Y. Wu,${ }^{6}$ W. Wu ${ }^{3}$ Y.C. Wu, ${ }^{1}$ Y.C. Wu,${ }^{4}$ Z.Z. Xianyu, ${ }^{1}$ H.Y. Xing, ${ }^{5}$ Y. Xu, ${ }^{3}$ \\ X.J. Xu ${ }^{1}$ T. Xue, ${ }^{1}$ L.T. Yang, ${ }^{1}$ S.W. Yang, ${ }^{7},{ }^{1}$ N. Yi,${ }^{1}$ C.X. $Y u,{ }^{3}$ H. Yu,${ }^{1}$ X.Z. Yu, ${ }^{5}$ X.H. Zeng, ${ }^{6}$ Z. Zeng, ${ }^{1}$ L. \\ Zhang, ${ }^{4}$ Y.H. Zhang, ${ }^{6}$ M.G. Zhao, ${ }^{3}$ S.N. Zhong, ${ }^{3}$ Z.Y. Zhou, ${ }^{2}$ J.J. Zhu, ${ }^{5}$ W.B. Zhu, ${ }^{4}$ X.Z. Zhu, ${ }^{1}$ and Z.H. Zhu ${ }^{6}$ \\ (CDEX Collaboration) \\ ${ }^{1}$ Key Laboratory of Particle and Radiation Imaging (Ministry of Education) \\ and Department of Engineering Physics, Tsinghua University, Beijing 100084 \\ ${ }^{2}$ Department of Nuclear Physics, China Institute of Atomic Energy, Beijing 102413 \\ ${ }^{3}$ School of Physics, Nankai University, Tianjin 300071 \\ ${ }^{4}$ NUCTECH Company, Beijing 10084 \\ ${ }^{5}$ School of Physical Science and Technology, Sichuan University, Chengdu 610065 \\ ${ }^{6}$ YaLong River Hydropower Development Company, Chengdu 610051 \\ ${ }^{7}$ Institute of Physics, Academia Sinica, Taipei 11529 \\ ${ }^{8}$ Department of Physics, Banaras Hindu University, Varanasi 221005
}

(Dated: October 1, 2018)

\begin{abstract}
The China Dark matter Experiment collaboration reports the first experimental limit on WIMP dark matter from $14.6 \mathrm{~kg}$-day of data taken with a $994 \mathrm{~g}$ p-type point-contact germanium detector at the China Jinping underground Laboratory where the rock overburden is more than $2400 \mathrm{~m}$. The energy threshold achieved was 400 eVee. According to the $14.6 \mathrm{~kg}$-day live data, we placed the limit of $\sigma_{\chi N}=1.75 \times 10^{-40} \mathrm{~cm}^{2}$ at $90 \%$ confidence level on the spin-independent cross-section at WIMP mass of $7 \mathrm{GeV}$ before differentiating bulk signals from the surface backgrounds.

PACS numbers: $95.35 .+\mathrm{d}, 98.70 . \mathrm{Vc}, 29.40 . \mathrm{Wk}$
\end{abstract}

There are many pieces of evidence from astroparticle physics and cosmology which indicate that about one quarter of the mass of our universe is composed of dark matter [1]. The nature of dark matter is unknown except that it is coupled with matter via gravity. One of the possible candidates for dark matter is the Weekly Interacting Massive Particles (WIMP, denoted by $\chi$ ) as motivated by many new theories beyond the standard model [2]. Direct detection of WIMP dark matter has been attempted with different detector technologies in the particle physics domain [3].

In recent years several experiments have expanded their coverage down to low mass WIMP with $m_{\chi}<10$ $\mathrm{GeV}$ [4 9]. A point-contact germanium detector can reach an energy threshold of hundreds of $\mathrm{eV}$ while keeping almost the same energy resolution as the traditional co-axial germanium detector [10]. Thus it can be a good choice for a low mass dark matter search. Based on our previous work [1], the China Dark matter Experiment (CDEX) collaboration has formally started a program aimed at the direct detection of low mass WIMP using a tonne-scale germanium array detector system [12]. As the first step, in 2011 the CDEX phase-I experiment (CDEX-1) started to test and run its first prototype p-type point-contact germanium (PPCGe) detec- tor with a crystal mass of $994 \mathrm{~g}$. The experiment took place in China Jinping underground Laboratory (CJPL) which was established at the end of 2010 . With $2400 \mathrm{~m}$ rock overburden, CJPL is the deepest operational underground laboratories for particle physics in the world. The cosmic-ray flux in CJPL is down to $61.7 \mathrm{y}^{-1} \mathrm{~m}^{-2}$ [13], and this makes it a very good site for ultra-low background experiments such as dark matter search, double beta decay, and so on.

The point-contact germanium detectors have also been used by several experiments [4, 9] to directly search for low mass WIMP. Due to the relative shallow cosmic-ray shielding, CoGeNT and TEXONO used muon-veto detectors to decrease the direct and indirect background contribution from cosmic-ray muons. The muon veto method can decrease the background contribution of the cosmic-rays, but is less efficient to pick out the background from secondary prompt and delayed cosmogenic radiation. The superior rock shielding of CJPL can minimize the cosmogenic influence. However it is still important to study the background of a prototype germanium detector to get more knowledge and experience for evaluating and developing a tonne-scale experiment with germanium detector.

In this article, we report the first results from the 
CDEX-1 experiment. The detailed information about the shielding system was described in reference [14]. This phase-I experiment did not install any active shielding system such as an anti-Compton detector in order to understand and estimate the background level of the PPCGe detector itself in CJPL, and it will helpful to compare and learn the background contribution of the anti-Compton detector and its veto efficiency installed in the next experiment phase. The passive shielding system was installed as, from outside to inside, $1 \mathrm{~m}$ of polyethylene, $20 \mathrm{~cm}$ of lead, $20 \mathrm{~cm}$ of borated polyethylene and $20 \mathrm{~cm}$ of oxygen-free high-conductivity copper.

Inside the cryostat of the PPCGe detector, the cylindrical germanium crystal has an $\mathrm{n}^{+}$type contact on the outer surface and a tiny $\mathrm{p}^{+}$type contact as the central electrode. A detailed illustration of the detector, electronics, and data acquisition (DAQ) system as well as its basic performances was also described in [14]. Signals from the $\mathrm{p}^{+}$electrode of the PPCGe were imported into a pulsed reset preamplifier which has 3 identical energyrelated outputs. Two of them were loaded to shaping amplifiers at $6 \mu \mathrm{s}\left(\mathrm{S}_{\mathrm{p} 6}\right)$ and $12 \mu \mathrm{s}\left(\mathrm{S}_{\mathrm{p} 12}\right)$ shaping time, respectively. The discriminator output of $\mathrm{S}_{\mathrm{p} 12}$ supplied the trigger for the DAQ. Another output was distributed to a timing amplifier $\left(T_{p}\right)$ to just amplify the pulse height of the raw-traces with few nano seconds shaping time. The pulsed reset preamplifier worked in pulsed mode, so it was reset to its initial condition by discharge the FET quickly marked by one reset inhibit signal. The charge and discharge procedure of the preamplifier was shown in Fig. 1(a) and the reset inhibit signals in Fig. 1(b). The reset inhibit signal was rectangular and quasi-periodic, and the period was typically around 0.7 second which was related to the leakage current of the PPCGe detector. Fig. 1(c) shows the output waveforms from the main amplifier with $12 \mu$ s shaping time. The discrimination output of the reset inhibit signal was also served as trigger to record the timing information of the discharging. Signals from the $\mathrm{n}^{+}$electrode of the PPCGe were read out by a resistive feedback preamplifier, and then were divided into 3 identical outputs followed by shaping amplifiers at $2 \mu \mathrm{s}$ shaping time but different gains. The $\mathrm{S}_{\mathrm{p} 6,12}, \mathrm{~T}_{\mathrm{p}}$ and $\mathrm{n}^{+}$electrode outputs were sampled and recorded by a $100 \mathrm{MHz}$ flash analog-to-digital convertor (FADC). The discriminator outputs of the random trigger events $(0.05 \mathrm{~Hz})$ from a pulse generator were served as trigger and digitized as well.

A first-run 18.1 day was taken, and the DAQ dead time was $0.5 \%$ as measured by the random trigger events. We focus in this paper on the analysis of this data set. Signals coming from $\mathrm{S}_{\mathrm{p} 12}$ were chosen as the energy measurement. We defined the pulse area of one event as its energy. The random trigger events were used for zeroing calibration. Rectangular pulser signals with various amplitudes were injected into the test input of the preamplifier of the PPCGe detector for trigger efficiency
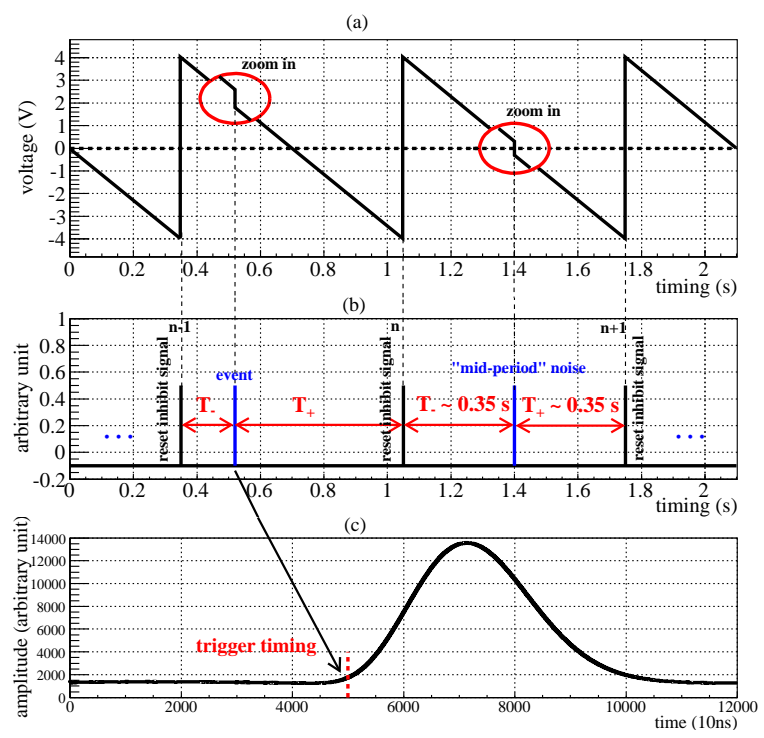

FIG. 1. Output of the PPCGe detector showing the relative timing: (a) raw signals from reset preamplifier; (b) timing of reset-inhibit and typical physics and "mid-period" noise events; (c) amplifier output with $12 \mu$ s shaping time.

\section{measurement.}

We used two kinds of methods to do the data selection and analysis. The first method is related to the timing information of one event. The second one is related to the characteristics of the pulse shape of one event.

In our experiment, noise events mostly occur in the middle range of the reset period while the physics events and the random trigger events are uniformly distributed. This kind of "mid-period" noise was due to the interference of the output of the preamplifier and the zero level when they are close to each other. As illustrated in Fig. 2(a) and (b), we denote $\mathrm{T}_{-}$as the time interval between the event and its nearest prior-inhibit signal, and $\mathrm{T}_{+}$the time interval between this event and its nearest post-inhibit signal. Fig. 2(a) shown the relationship of $\mathrm{T}_{-}$and $\mathrm{T}_{+}$for both random trigger events and background events. These can therefore be rejected by the "TT Cut" which leads to a live time penalty. The background spectra before and after the TT cut, as well the random trigger spectra, have been shown in the Fig. 2(b, c).

The pulse shape discrimination is based on two methods. The first method is the "pedestals of $\mathrm{S}_{\mathrm{p} 6,12}$ and $\mathrm{T}_{\mathrm{p}}$ " (Ped) cuts which are applied to discriminate those events whose pedestals exhibit anomalous behaviour. The criteria are derived from the random trigger events whose pedestal distributions are considered consistent with the physical events. The signal efficiencies for the TT and Ped cuts, which are independent on the pulse amplitude, were accurately measured with random trigger events, and are $92.5 \%$ and $96.2 \%$, respectively. A data set of $16.0 \mathrm{~kg}$-day has been obtained after TT and Ped cuts. 

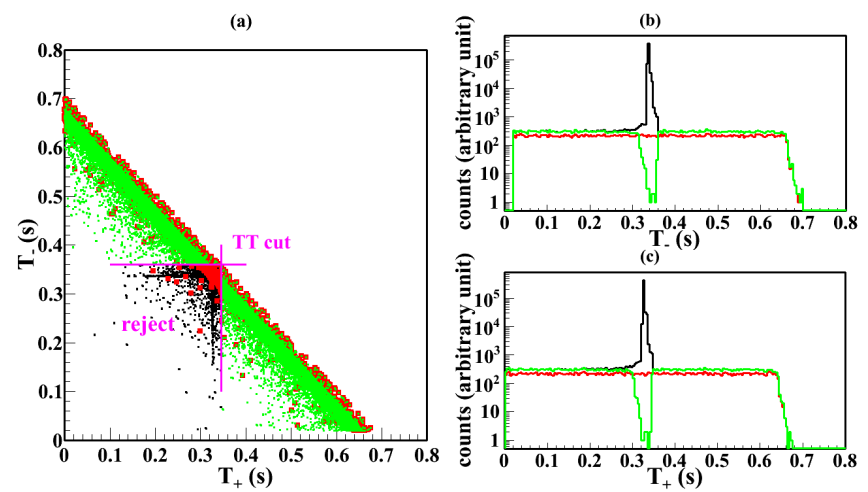

FIG. 2. The scatter plots of $T_{+}$and $T_{-}$for random trigger events (red color) and background events before and after the TT cut (black and green color) have been shown in Fig. 2(a). The TT cut has also been overlaid on the scatter plot. The spectra before and after the TT cut have been given in the Fig. 2(b, c), at the same time, the $\mathrm{T}_{+}$and $\mathrm{T}_{-}$spectra for random trigger have also been shown in these figures.

The second method is the pulse shape discrimination (PSD) cuts which are effective approaches to reject remaining noise events, including one cut based on the correlation between the integration area and its maximum amplitude of $\mathrm{S}_{\mathrm{p} 12}$, and another cut based on the correlation between signals from $\mathrm{p}^{+}$electrode and $\mathrm{n}^{+}$electrode for one event. The efficiencies of the PSD cuts were measured by a ${ }^{241} \mathrm{Am}$ source sample and a ${ }^{137} \mathrm{Cs}$ source sample $\left(\varepsilon_{\mathrm{PSD}}\right)$. The spectra of ${ }^{241} \mathrm{Am}$ source along the analysis chain are given in Fig. 3(a). The efficiencies derived from ${ }^{241} \mathrm{Am}$ and ${ }^{137} \mathrm{Cs}$ spectra agree very well and the bin-by-bin combined $\varepsilon_{\mathrm{PSD}}$ are shown in Fig. 3(b). The source spectra provide consistent measurements of the TT and Ped cuts efficiencies to those derived from random trigger events.

The distribution of the random noise are given in Fig. 3(b). The energy resolution at this zero energy point is 51 eVee ("ee" represents electron equivalent energy). The hardware discrimination threshold, defined at $50 \%$ trigger efficiency, is calculated about $343 \mathrm{eVee}$ and much higher than the $5 \cdot \sigma(255$ eVee). In addition, the trigger efficiency is nearly equal to $100 \%$ above 500 eVee.

Considering both the trigger and signal selection efficiencies, the 400 eVee energy threshold is selected as our energy threshold for physical analysis. Fig. 4(a) shows the low energy background spectrum detected by the PPCGe detector with corrections of the $\varepsilon_{\text {trig }}$ and cuts efficiencies, fiducial mass and the dead time. Both statistic and systematic errors are considered with standard error propagation. Several characteristic X-ray peaks can be seen. They are due to the cosmogenic radioactive isotopes which are mainly generated within the germanium crystal before installation into CJPL, and include ${ }^{68,71} \mathrm{Ge},{ }^{68} \mathrm{Ga},{ }^{73,74} \mathrm{As},{ }^{65} \mathrm{Zn}$, and so on. Because of the $1.5 \mathrm{~mm}$ oxygen-free high-conductivity Copper cryostat
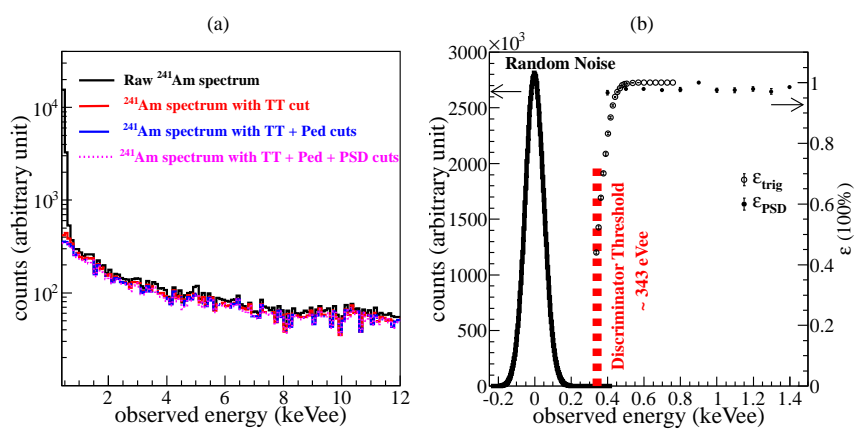

FIG. 3. The ${ }^{241}$ Am spectra along the analysis chain are shown in Fig. 3(a). The distribution of the random noise fluctuation and the discriminator threshold of our hardware are given in Fig. 3(b). The trigger efficiency $\varepsilon_{\text {trig }}$ and PSD cuts efficiency $\varepsilon_{\mathrm{PSD}}$ have also been shown in the same plot.
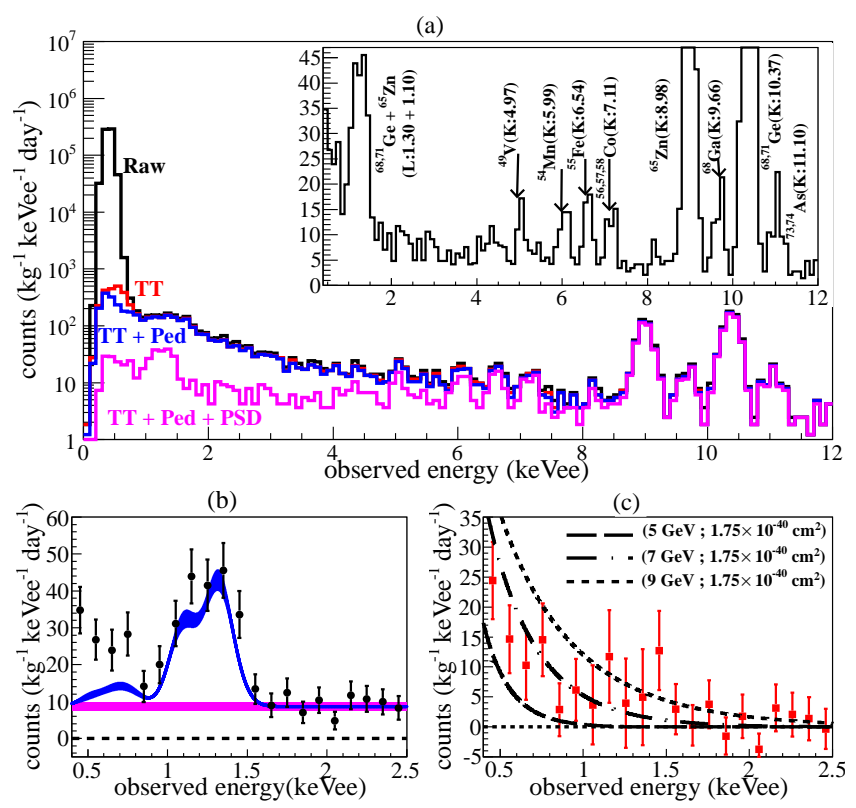

FIG. 4. The observed energy spectra showing raw data, data after TT cut, TT + Ped cuts and TT + Ped + PSD cuts were given in Fig. 4(a), respectively. The inset plot in Fig. 4(a) showed the background spectrum after TT + Ped + PSD cuts with both the $\varepsilon_{\text {trig }}$, cuts efficiencies, fiducial mass, and the dead time correction. Eight $K$-shell peaks for $L$-shell peaks prediction are identified. The low energy spectrum in the range of $0.4-2.4 \mathrm{keVee}$ was shown in Fig. 4 (b), as well the calculated $L$-shell background contribution and the flat $\gamma$ background with the expanded statistical error band. The residual spectrum was shown in Fig. 4 (c) superimposed with the predicted spectra for $5 \mathrm{GeV}, 7 \mathrm{GeV}$, and $9 \mathrm{GeV}$ WIMP with spin-independent cross-section $\sigma_{\chi N}=1.75 \times 10^{-40} \mathrm{~cm}^{2}$.

surrounding the germanium crystal, the external low energy x-rays cannot enter into the bulk of the PPCGe detector. Energy calibration was therefore accomplished by using these internal origin radioactive isotopes. The decays of the $K$-shell $(10.37 \mathrm{keV})$ and $L$-shell $(1.29 \mathrm{keV})$ 
peaks of ${ }^{68,71} \mathrm{Ge}$ isotopes accord well with their expected half-life [14]. As the characteristic x-rays are internal and short-ranged, the detection efficiency is almost $100 \%$. The ratios of $K$-shell to $L$-shell X-ray events calculated based on reference [15] are used to predict the intensity of $L$-shell in the lower energy ranges $(<2 \mathrm{keVee})$. The background spectrum in the low energy range of 0.4 2.4 keVee and the $L$-shell contributions calculated from the eight clearly visible $K$-shell peaks has been shown in Fig. 4(b).

We do not apply the surface-bulk cut in this analysis. Accordingly, from simulations and previous measurements [4, 6, 9], there should be a flat $\gamma$ spectrum contributed by the bulk $\gamma$ events which is mainly located at the internal volume of a PPCGe detector and monotonously decreasing background spectrum from anomalous surface events due to incomplete charge collection. so that the expected background should be monotonously decreasing. In addition to the L-shell Xrays contributions, a conservative flat background level was subtracted at an energy range beyond the tails of the $\chi-\mathrm{N}$ nuclear recoil spectrum which, for $m_{\chi}<12 \mathrm{GeV}$, corresponds to 1.7-2.4 keVee. The final residual spectrum in the region of $0.4-2.4$ keVee is shown in Fig. 4(c) from which the constraints on WIMP are derived.

The thickness of the outer $\mathrm{n}^{+}$layer of a $\mathrm{p}$-type germanium detector can be measured by a multi $\gamma$-ray isotope, such as ${ }^{133} \mathrm{Ba}$ [16]. Due to the close match in total mass and the structure between the CDEX-1 and TEXONO [9] detectors, we chose the same depth of the dead layer as $1.16 \mathrm{~mm}$ with an uncertainty of $10 \%$ for easy comparison. This gives rise to a fiducial mass of $905 \mathrm{~g}$ with an uncertainty of less than $1 \%$, corresponding to a data size of $14.6 \mathrm{~kg}$-day.

The quenching factor of the recoiled Ge nucleus is given by the TRIM program [17]. The parameters chosen for the WIMP in thermal equilibrium includes Maxwellian velocity distribution with $\nu_{0}=220 \mathrm{~km} \cdot \mathrm{s}^{-1}$, the escape velocity $\nu_{\mathrm{esc}}=544 \mathrm{~km} \cdot \mathrm{s}^{-1}$ and the local density $\left(\rho_{\chi}\right)$ of $0.3 \mathrm{GeV} \cdot \mathrm{cm}^{-3}$. The energy resolution of the PPCGe was derived from the calibration data and then extrapolated to the region less than $1 \mathrm{keV}_{\mathrm{ee}}$.

The predicted spectrum of WIMP-nucleon spinindependent interaction can be evaluated. Using the standard WIMP halo assumption [20], the light-WIMP spectra corresponding to $5 \mathrm{GeV}, 7 \mathrm{GeV}$ and $9 \mathrm{GeV}$ WIMP with spin-independent cross-section $\sigma_{\chi N}=1.75 \times$ $10^{-40} \mathrm{~cm}^{2}$ are also put on the spectrum in Fig. 4(c).

Assuming all of the events from our final residual spectrum are induced by incident WIMP, we can derive upper limits on the WIMP-nucleon spin-independent cross section at different WIMP masses. Binned Poisson method [21] is utilized and the exclusion curve with $90 \%$ C.L. is displayed in Fig. 5, along with the results from other experiments [4 8, 18]. Although we did not apply the bulk-surface cut and the anti-Compton suppression, the

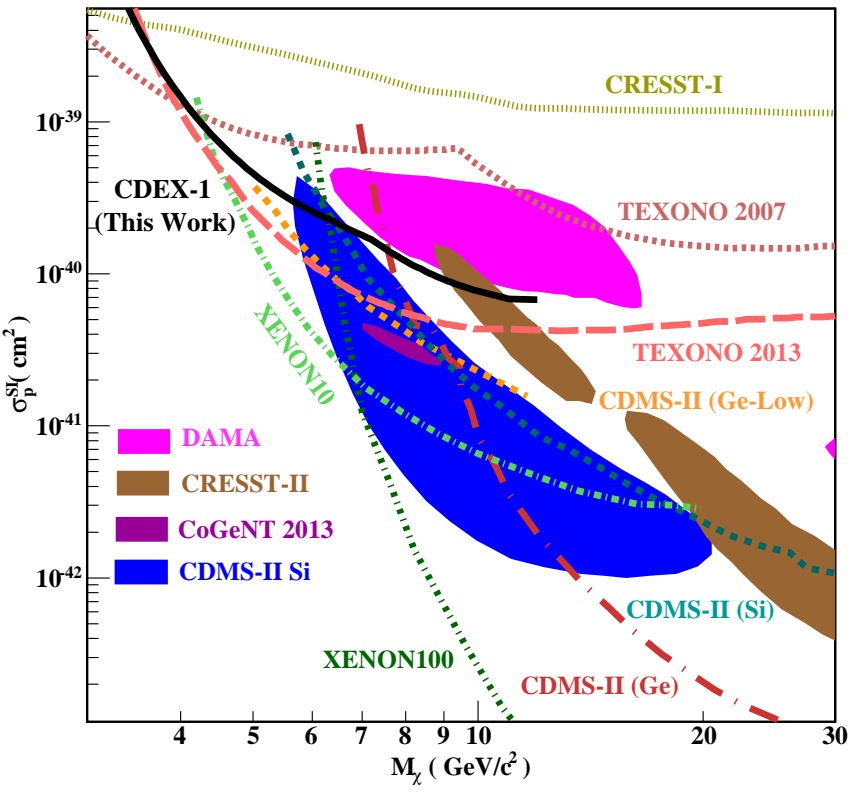

FIG. 5. Exclusion plot of spin-independent coupling, superimposed with the results from other benchmark experiments. The results is also shown in this figure including the $90 \%$ confidence regions favored by CoGeNT [4], DAMA/LIBRA [5], and CRESST-II [18], as well the exclusion limits from CDMS-II (Si) 6], XENON100 and the low-threshold analysis of XENON10 [8], TEXONO [9], and CRESST-1 [19].

results are close to the latest sensitivities of the TEXONO experiment 9]. The bulk-surface cut could reduce the background level by a factor of 2-3 [4, 9] and we expect that our new result with bulk-surface cut can be used to check these results. Also the residual spectrum we have achieved need to be understood further with more data.

An anti-Compton detector will be added to test its performance and background level in the regime of low cosmic-ray flux. It will need to be evaluated whether the suppression power of this anti-Compton detector in CJPL can balance its additional contribution to the PPCGe's background due to its own radioactivities in detail. This will aid in the understanding of the cosmicray induced background when compared with the results from the reference [9], and it will be very helpful for the evaluation of the sensitivity of the possible future tonnescale germanium experiment in the dark matter search by CDEX collaboration.

This paper represents the first scientific results obtained at the new underground facilities CJPL. The authors would like to thank all those who contributed to its efficient construction and commissioning. We are grateful to X. Q. Li and Y. F. Zhou for useful comments. This work was supported by the National Natural Science Foundation of China (contract numbers: 10935005, 10945002, 11275107, 11175099) and National Basic Research program of China (973 Program) (contract num- 
ber: 2010CB833006).

* Corresponding author: yueq@mail.tsinghua.edu.cn $\dagger$ Participating as a member of TEXONO Collaboration

[1] J. Beringer et al., Phys. Rev. D 86, 010001 (2012), and references therein; P. A. R. Ade et al., (2013), arXiv:1303.5076v1,

[2] C. Kelso, D. Hooper, and M. R. Buckley, Phys. Rev. D 85, 043515 (2012), and references therein.

[3] J. D. Lewin and P. F. Smith, Astropart. Phys. 6, 87 (1996)

[4] C. $\quad$ E. Aalseth et al., Phys. Rev. Lett. 101, 251301 (2008) C. E. Aalseth et al., Phys. Rev. Lett. 106, 131301 (2011) C. E. Aalseth et al., Phys. Rev. Lett. 107, 141301 (2011). Phys. Rev. D 88, 012002 (2013).

[5] P. Belli et al., Phys. Rev. D 84, 055014 (2011) R. Bernabei et al., Eur. Phys. J. C 67, 39 (2010).

[6] Z. Ahmed et al., Science 327, 1619 (2010); Z. Ahmed et al., Phys. Rev. Lett. 106, 131302 (2011); Z. Ahmed et al., (2012), arXiv:1203.1309 R. Agnese et al., (2013), arXiv:1304.3706v2 R. Agnese et al., „(2013), arXiv:1304.4279v2.
[7] G. Angloher et al., Eur. Phys. J. C 72, 1971 (2012)

[8] E. Aprile et al., Phys. Rev. Lett. 107, 131302 (2011) J. Angle et al., Phys. Rev. Lett. 107, 051301 (2011) E. Aprile et al., Astropart. Phys. 35, 573 (2012) E. Aprile et al., Phys. Rev. Lett. 109, 181301 (2012).

[9] S. T. Lin et al., Phys. Rev. D 79, 061101 (2009) H. B. Li et al., Phys. Rev. Lett. 110, 261301 (2013)

[10] P. N. Luke et al., IEEE Trans. Nucl. Sci. 36, 926 (1989)

[11] Q. Yue et al., High Energy Phys. Nucl. Phys. 28, 877 (2004); D. He et al., High Energy Phys. Nucl. Phys. 30, 548 (2006); X. Li et al., High Energy Phys. Nucl. Phys. 31, 564 (2007).

[12] K. J. Kang et al., J. Phys. Conf. Ser. 203, 012028 (2010) Q. Yue and H. T. Wong, J. Phys. Conf. Ser. 375, 042061 (2012)

[13] Y. C. Wu et al., Chinese Phys. C 37, 086001 (2013).

[14] K. J. Kang et al., ${ }_{\square}(2013)$, arXiv:1305.0401.

[15] J. N. Bahcall, Phys. Rev. 132, 362 (1963).

[16] E. Aguayo et al., „2012), arXiv:1207.6716.

[17] J. F. Ziegler, Nucl. Instrum. Methods Phys. Res., Sect. B 219-220, S. T. Lin et al., (2007), arXiv:0712.1645v4

[18] M. Felizardo et al., Phys. Rev. Lett. 108, 201302 (2012) S. Archambault et al., Phys. Lett. B 711, 153 (2012)

[19] G. Angloher et al., Astropart. Phys. 18, 43 (2002)

[20] F. Donato, N. Fornengo, and S. Scopel, Astropart. Phys. 9, 247 (1998)

[21] C. Savage et al., J. Cosmol. Astropart. Phys. 2009, 010 (2009) 